\title{
Pose and Pathosformel in Aby Warburg's Bilderatlas
}

\author{
Leonardo Impett, Sabine Süsstrunk \\ School of Computer and Communication Sciences, \\ École Fédérale Polytechnique de Lausanne, Switzerland \\ leonardo.impett@epfl.ch
}

\begin{abstract}
We look at Aby Warburg's concept of Pathosformel, the repeatable formula for the expression of emotion, through the depiction of human pose in art. Using crowdsourcing, we annotate 2D human pose in one-third of the panels of Warburg's atlas of art, and perform some exploratory data analysis. Concentrating only on the relative angles of limbs, we find meaningful clusters of related poses, explore the structure using a hierarchical model, and describe a novel method for visualising salient characteristics of the cluster. We find characteristic pose-clusters which correspond to Pathosformeln, and investigate their historical distribution; at the same time, we find morphologically similar poses can represent wildly different emotions. We hypothesise that this ambiguity comes from the static nature of our encoding, and conclude with some remarks about static and dynamic representations of human pose in art.
\end{abstract}

Keywords: Pose, Pathos, Emotion, Pathosformel, Bilderatlas, Warburg

\section{Introduction}

Aby Warburg's Bilderatlas, an unfinished atlas of the history of art, consists of 1230 photographs of various kinds (styles, periods and media) of art, arranged in 63 panels. Warburg wrote little explicitly about the Bilderatlas (and he died before it could be completed); but we do understand that Warburg was interested in two key concepts, Pathosformel (formulas that express emotion), and the Nachleben der Antike. The panels of the Bilderatlas have been studied extensively by art-historians and iconographers, often cross-referencing the seemingly ambiguous panels with Warburg's extensive previous work (see e.g. [3]). Excellent introductions to Warburg and to the Bilderatlas exist elsewhere (see eg. Foster's preface in [24], [19]); here, we content ourselves with a brief introduction of two key Warburgian concepts mentioned above.

\subsection{The Pathosformel}

The Pathosformel (plural Pathosformeln), or formula of pathos (emotion), is a key Warburgian concept. On the most basic level, it describes the portrayal or communication of emotion, movement and passion, through a repeatable visual 
paradigm or formula. Salvatore Settis describes it as: "the oxymoronic word, in that it merges in the same term the movement of pathos and the rigidity of the formula-schema" [13, p. 167]. Freedberg [14] notes the intuitive nature of the Pathosformel - asking "How does a picture or sculpture engage the body, and what are the emotional responses that may ensue?... Not only do the gestures ring humanly true, one also knows immediately how often one has seen them in art".

We understand these Pathosformeln specifically as body poses, neglecting other portrayals of emotion (such as accessory items, facial expression and hand gestures) - as human pose is both a formally quantifiable aspect of a work of art, and a primary expression of emotion (as further described in Section 2.2).

\subsection{Nachleben der Antike}

Warburg theorised that classical Pagan formulae (from Greek and Roman times), particularly in the portrayal of elementary impulses, were reborn in the Italian Renaissance. He called this the Nachleben der Antike, the after-life of classical antiquity [24].

Warburg's collaborator and assistant, G. Bing, noted in particular the emotional strength of art in antiquity: "The gestures of classical art, in their first formulations, come from a period in which the reality of myths was a ritual reality. . . These gestures are still capable of provoking a suitable reaction" 1 .

These antique formulae were not rediscovered in an archeological sense, but rather preserved in common cultural memory. They fell out of favour during the medieval period, as the "expression of elementary impulses" [24] was prohibited for religious reasons. When Pathosformeln are renewed in the Renaissance, the emotion or meaning attributed to a certain formula often changes - the so-called 'antithetical' principle (see [24, p. 38]).

We will seek to describe formally the Nachleben of Pathosformeln through the Bilderatlas. Indeed, the entire Bilderatlas has been described as "a means to chart the afterlife of ancient forms through time to his present day" [8].

\section{Emotion as pose}

\subsection{Within art history}

Human form has long been part of art-historical analysis, particularly in sculpture - see for example Argan's comparison of syntactic (each human body separately) and paratactic (the relationship between bodies) analysis of pose [4, p. 49]. Analysis of classical sculpture includes such concepts as the contrapposto, where the weight of the statue is supported by one foot - twisting the axis of the hips and legs [4]. More recently, Robin Osborne's monograph [20] has attempted to describe social and historical aspects of the history of art through the depiction of bodies in Greek antiquity.

\footnotetext{
${ }^{1}$ All translations by the author
} 
David Freedberg, an art historian and current director of the Warburg Institute, makes three specific claims that are relevant to our analysis [14]:

1. We can draw connections between art (as images) and the perception of art on the level of emotions, feeling and empathy

2. Emotions might be classifiable (as suggested by Ekman [11])

3. It is impossible to consider emotions as separate from the body, or indeed from the movement of the body

The first two claims form good art-historical assumptions for our study of Pathosformeln through human pose; the third, hinting at movement, lies at the base of our proposed future work. Warburg's interest in motion within images has recently explored in detail by Philippe-Alain Michaud [19].

\subsection{Psychological and psychophysical evidence}

Most experimental evidence for the emotional perception of pose follows the Light Spots Model of Johansson (1973) [16]. Instead of a whole video or image of a person, only some bright spots are shown (lights fixed to the body), describing the position of the main joints of the body. Johansson found that between 10 and 12 such spots are adequate for 'a compelling impression of human walking, running, dancing etc.'. These Light Spots are therefore a compelling analogue of our own reduced pose model for paintings (our own analysis, described subsequently, uses 12 points to describe a human figure).

The most important results from the psychological literature seem to focus on the difference between static pose (photographs) and dynamic pose (videos). Recently, it has been shown that dynamic pose conveys qualitative ('which emotion?') as well as quantitative ('how intense?') information [5]. This echos the much more recent results found in action classification for computer vision, which - although not concerned with emotion recognition - can classify actions much more successfully with dynamic data [21].

Static pose, when seen as a single frame of a dynamic sequence, leads to emotional ambiguities. Indeed Atkinson found that, if we reverse the time-direction of Light Spots videos of actors, the percieved emotion changes. This, it is argued, is evidence that static form (pose) and dynamics give distinct contributions to the perception of emotions [6]. This inherent ambiguity in static pose may not be inconsistent with Warburg's own writings on the antithetical nature of the Nachleben.

From this, it could be suggested that artists use visual cues of movement to disambiguate between the different possible emotive implications of the same pose. In the analysis presented in the rest of this report, we focus only on static pose - whilst being conscious of the additional ambiguities presented by this reduction.

Our study explicitly ignores facial expressions, which are well-studied by psychologists; it has been suggested [7], however, that body cues play the major role in the perception of emotions from photographs, substantially informing our interpretation of facial expressions. 


\section{Collecting a dataset}

In keeping with our understanding of the Pathosformel as human pose, we have started to create a dataset of the pose of every figure in the Bilderatlas. Although our dataset is created with an analysis of Nachleben and Pathosformel in mind, it is also intended to serve as the basis for a future training dataset for automatic pose detection in paintings, currently an unsolved problem (see for example [15], $[9])$.

\subsection{Digitising the Bilderatlas}

As the panels of the Bilderatlas no longer exist, our only primary digital sources are high-resolution ${ }^{2}$ scans of the 1920s black-and-white photographs of the Bilderatlas. Unfortunately, even high-resolution scans of Panels are frequently not of high enough quality to be able to discern smaller individual bodies clearly.

For this reason, colour photographs of the original artwork were sourced for 21 of the Bilderatlas' 63 panels. The originals were mainly collected from museum websites and open-access art collections. Images that clearly contained no human figures (such as unillustrated manuscript pages) were not sourced. 9\% of the images were either not found, or contained no human bodies. We have 318 images for our 21 panels from this process, which is $26 \%$ of the total number of works in the Bilderatlas (1230).

\section{$3.2 \quad$ Two-stage annotation}

We crowdsourced the annotation of poses in our images using the CrowdFlower platform [23]. This platform uses its own workers, as well as those of other services (such as Amazon Mechanical Turk and SamaSource) - see [12] for a detailed comparison with Amazon Mechanical Turk.

After a series of preliminary experiments on crowdsourced pose annotation, we designed a two-stage system for the annotation of poses in paintings. In particular, we found that when asked to annotate poses directly (given a whole painting):

1. Individual bodies are often too small to be annotated accurately on-screen

2. We often find groups of people standing close to one another in paintings; these can make the pose annotation of each body difficult and confusing

3. When paid per painting, the worker has an economic motivation to not annotate all the bodies

4. If users decide to annotate a different subset of the people in a painting, annotations cannot be aggregated.

We therefore split the pose annotation task into two stages (see Figure 1):

1. Individual bodies are segmented from the paintings

${ }^{2}$ Some of the panels are digitsed to size $3000 \times 4030$ pixels 
2. Each pose is annotated separately from a cropped figure of that body

By giving a smaller, cropped figure containing the relevant body, annotation becomes clearer and more explicit for the user, and pose is more easily aggregated.

We should acknowledge at this point that some paintings will have many tens of figures (such as battle scenes); others will have just one. Our decision to annotate all figures in each painting gives a bias in our dataset towards those paintings with more figures.

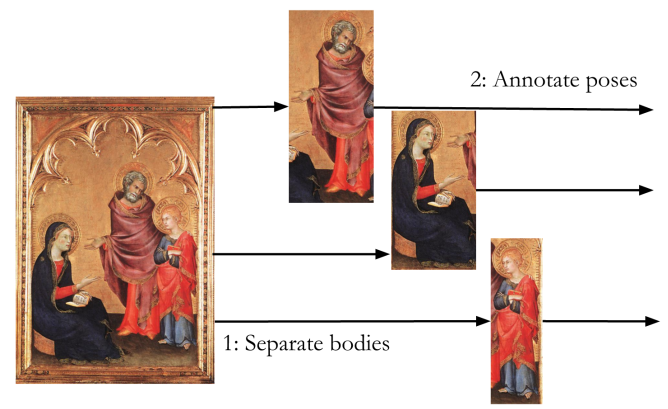

Fig. 1: A diagram of our two-stage pose annotation process

\subsection{Aggregation algorithms}

Extracting bodies In order to extract bodies from the paintings, crowd workers were asked to draw a line from the face to the foot of each figure. This gives an indication of both position and size of the body within the painting. Whilst the annotation of feet varies widely (for instance, when the feet of a figure are spread), the annotation of the head is precise, and gives us an explicit 2D point for matching separate annotations.

Each painting was annotated separately by three annotators - giving three separate sets of annotations. Aggregation was performed on the annotations in the following way:

1. Pick the set of annotations with the most lines (ie, the most bodies)

2. Keep the annotations that agree with at least one other set (where the heads are within a 50 pixel radius)

3. Construct circles around the annotations, using the head-foot lines as tangents (giving an area for the body, even where the lines are horizontal)

4. Crop $1.5 \times$ the width and height of this area

Note that we never average the aggregation, only compare agreement - it was found that averaging between clustered annotations can give artefacts (eg. where 
heads of different bodies are close together). Failure cases include non-human bodies being reliably annotated (Figure $2 \mathrm{~d}$ ), and incomplete bodies (where pose is not fully defined) (Figure 2e) - both cases are rare.

We often find that more than one body is included in the final crop - for this reason, we also display the labelled points of the head and foot of the relevant body (see Figure $2 \mathrm{~b}$ ).

In total, we extract 1,772 figures of individual bodies, from our 318 original images.

Single human pose Once figures of individual bodies had been produced, the pose of each body was annotated - again using three separate annotations. This task involved drawing twelve lines (implying 24 points), corresponding to the twelve limbs of the figure (see Figure 3a). Compared to datasets on photographic pose estimation (such as the Leeds Sports dataset [18]), we omit the hips, as they were often visually ambiguous in the paintings and (in our preliminary experiments) frequently omitted by workers.

Due to the fact that we sometimes have more than one figure in the cropped image (see Figure 2b), we eliminate cases where the pose of the wrong person seems to have been annotated. We reject annotations where the average of all limb co-ordinates (the centre of mass of the body) is more than one spine-length away from the average of all the other annotations.

We then take the average skeleton. The standard deviation of the individual (non-rejected) annotations around the average, when normalised by the spine length of each body (to compare bodies of different size), is 0.23 . Overall, 1665 aggregate annotations were produced from our 1773 skeletons (94\%) - with the remaining $6 \%$ of figures not having any accepted annotations (eg. due to the failure cases shown in Figure 2d and 2e).

\section{Data analysis}

After annotating and aggregating our human poses, we perform a series of normalisations on the data before our unsupervised learning models:

1. All limb lengths are ignored, and only the angles of limbs are conserved

2. All bodies are rotated such that their spine is vertical

3. Poses are mirrored such that their highest arm is on the right

4. The angles of the lower body are multiplied, such that the sum of the variances in upper and lower body angles is equal (due to anatomical constraints, variance in lower body angles is always smaller than that in the upper).

Our 12 2-dimensional co-ordinates (for each pose) thus become an 11-dimensional angular vector. 


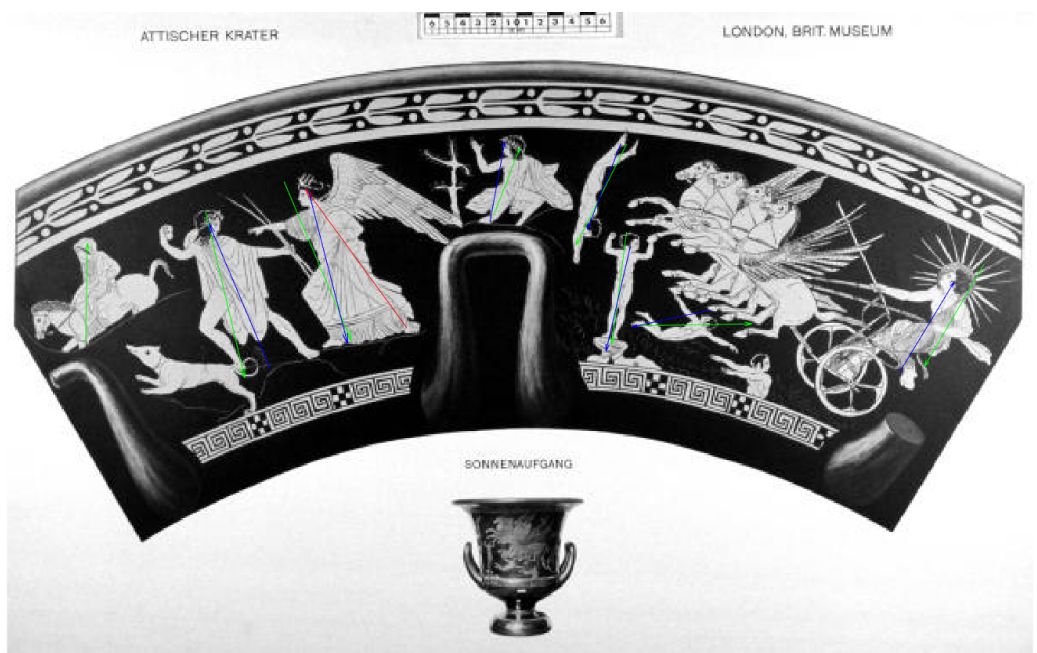

(a) A set of annotations for Adolf Furtwängler's copy of a 5th century BC Puglian vase - Figure 1 from Panel 2 of the Bilderatlas. The coloured arrows show three complete sets of annotations (red, blue and green).

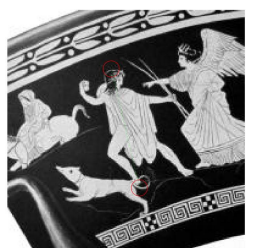

(b) Successful segmentation from (a)

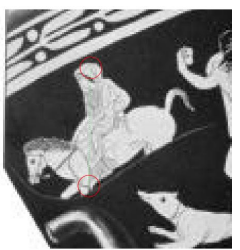

(c) Successful segmentation from (a)

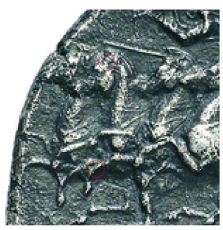

(d) Failure case; non-human body

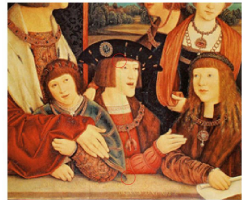

(e) Failure case; incomplete body

Fig. 2: Finding bodies in images - annotations (a), successful segmentations (b-c) and failure cases (d-e). Note in (a)-(c) how the green annotator has identified all bodies correctly; the blue missed a few, and the red has only indicated a single body. This variance in the number of bodies annotated is a good reason to split pose annotation into two parts; to make sure the same poses are annotated each time. 


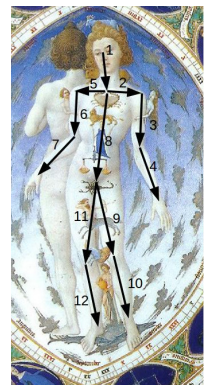

(a) The twelve lines of our pose annotation - used in the instructions for crowdworkers

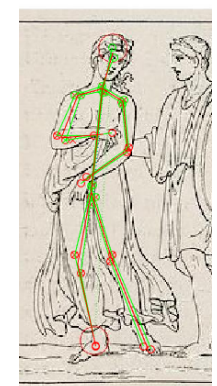

(b) An example aggregate body pose (in red), showing individual annotations (in green)

Fig. 3: Body pose annotation - our second-stage crowdsourcing

\subsection{Unsupervised learning}

Hierarchical clustering In order to compare human poses in the Bilderatlas morphologically, we seek to build hierarchical trees of poses from our dataset.

Hierarchical clustering works well in non-Euclidian spaces, as requires only a point-to-point distance metric. For our purposes, we define the distance between pose $i$ and $j$ as:

$$
D\left(P_{i}, P_{j}\right)=\sum_{k=1}^{11}\left\|\theta_{i, k}-\theta_{j, k}\right\|_{2}
$$

We use agglomerative hierarchical clustering - each data-point starts in its own cluster, and we merge the two clusters with the smallest pairwise distance $D$, until all the data is in one cluster. The order of these merging operations gives us a hierarchical tree, which we can represent in a dendrogram (see Figure 4).

Two-stage clustering Hierarchical clustering can give us a good impression of morphological structure between similar poses, such as those in Figure 4. The higher-level structure, however, is less meaningful - groups of completely separate poses (running, sitting, walking) have no intuitive hierarchical relationship between them.

For this reason, we attempt first to cluster general types of poses. We use a two-stage clustering technique:

1. Different pose-types are defined using K-means clustering

2. Within each pose-type, variations and structure is investigated using hierarchical clustering 


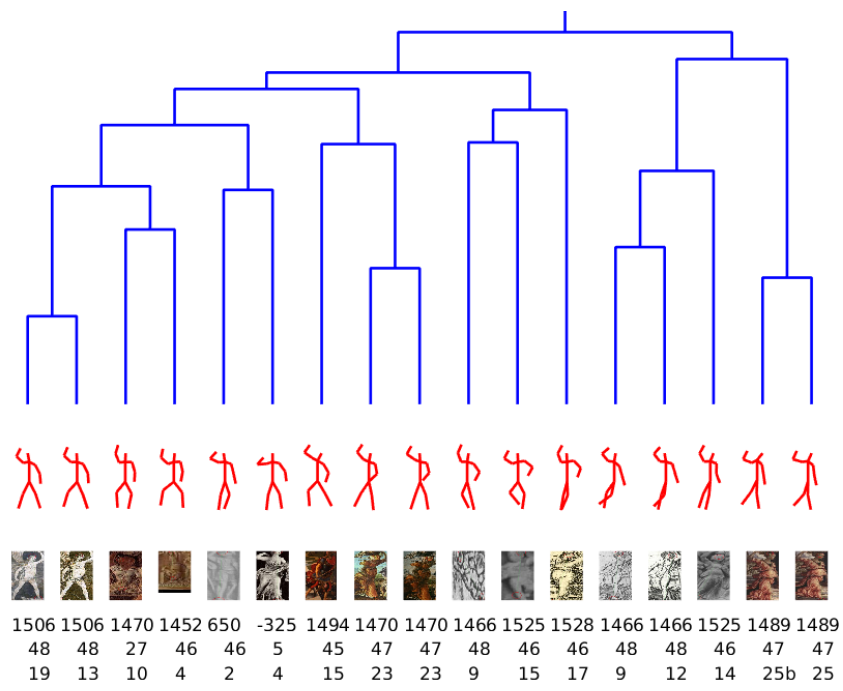

Fig. 4: A detail from a dendrogram of poses from our dataset, showing the pose, original image, year, panel number and image index for each pose.

K-means with stereographic projections Unlike hierarchical clustering, K-means cannot be applied naïvely to angular data - it cannot encode angle wraparound. Although appropriate variations of K-means exist, we follow Dortet $\&$ Berdanet [10] in simplifying the problem using stereographic projection. Here, for each person $i$, we map each angle $\theta_{k}$ to Cartesian form $\left(x_{k}, y_{k}\right)$. It can be shown that the mean in stereographic space is equivalent to the circular mean in angular space.

We apply a K-means algorithm (with 50 replicates) to our pose-data in stereographic space. After manual inspection of the cluster means, original figures and associated in-cluster variances for a range of $K$ from 2 to 50, we choose to learn $K=16$ clusters.

\subsection{Visualisation}

For an art-historical interpretation of the clusters, intutive and accurate visualisations are required. Plotting the cluster mean is uninformative of the variance of each cluster - and can also lead to an uncharacteristically static pose (when compared with the cluster dendrograms).

We therefore model each cluster (in stereographic space) as a Gaussian, with mean $\mu$ and covariance $\Sigma$. To visualise the range of skeletons, we then take 10 samples from a narrower Gaussian: $\theta \sim N(\mu, 0.1 \times \Sigma)$.

Figure 5 shows the result of this Gaussian-sample visualisation. The range of each limb gives a qualitative, relative impression of the variance in each angle, whilst the silhouette of the figure gives an impression of the mean. We can 
immediately spot clusters with a very large variance (such as Cluster 10), as well as much narrower ones (Cluster 1).

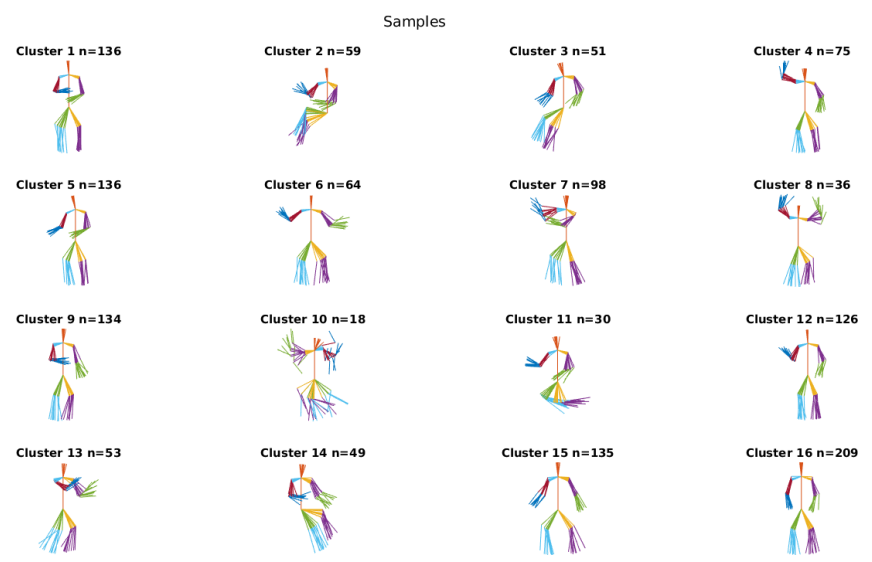

Fig. 5: A visualisation of 10 Gaussian samples taken from each cluster, showing the relative variance of each limb, for each cluster.

\subsection{Ideal-type}

Weber and the Ideal-Type Although our Gaussian-sample visualisations give an idea as to the range of poses present in each cluster, it is interesting (from an art-historical perspective) to ask a subtly different question: what are the defining characteristics of each cluster? For instance, Christopher Johnson - in analysis of the figure of the Nymph - notes that [1, p.105] "The nymph is the paradigm of which individual nymphs are the exemplars". For each cluster, we seek to identify this paradigm.

We interpret this set of defining characteristics as a Weberian ${ }^{3}$ ideal-type ${ }^{4}$ : the goal of ideal-type concept-construction is always to make clearly explicit not the class or average character but rather the unique individual character of cultural phenomena [25, p. 101-102].

We understand this ideal-type as a frequentist hypothesis-testing paradigm, where we test the hypothesis that each angle $\theta$ is differently-distributed for a single cluster than it is for the whole dataset. A detailed discussion on the deeper parallels between Weberian ideal-types and hypothesis-testing scientific models is provided elsewhere [26].

${ }^{3}$ Weber and Warburg knew and respected each other's work [22, p. 68-69]

${ }^{4}$ Note that Weberian ideal-types are explicitly not 'ideal' in a value-judgement sense. 


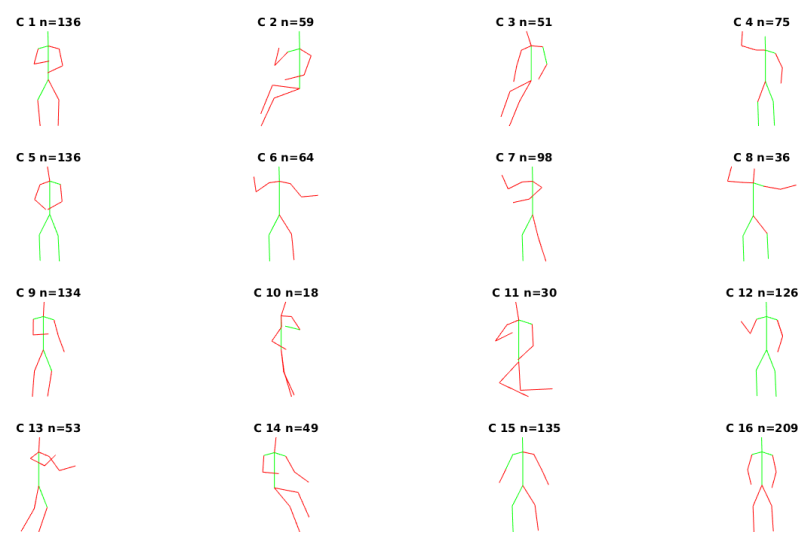

Fig. 6: Our frequentist ideal-type visualisation - limbs which compromise defining characteristics are coloured in red.

Frequentist interpretation In order to identify the defining characteristics of each cluster, we apply a two-sample Kolmogorov-Smirnov test for the distributions of each angle $k$, for each cluster $i$. The null hypothesis of this test is that samples were generated from the same distribution. Under our understanding of the ideal-type, if we can reject the null hypothesis, we can define the angle $k$ as a defining characteristic of cluster $i$. In other words, if the distribution of ${ }_{i, k}$ is significantly different to ${ }_{k}$ (for all $i$ ), then the limb $k$ is a defining characteristic of cluster $i$.

The Kolmogorov-Smirnov statistic between two samples, $\theta_{1}$ and $\theta_{2}$, is defined as:

$$
D=\max _{x}\left(\left|F_{1}(x)-F_{2}(x)\right|\right)
$$

Where $F_{1}(x)$ is the proportion of $\theta_{1}$ values that are less than $x$. We reject the null hypothesis (two samples are drawn from the same distribution) when:

$$
D>c(\alpha) \frac{n+n^{\prime}}{n n^{\prime}}
$$

at some confidence level $\alpha$ - we use a lookup table to calculate $c(\alpha)$. We use a $95 \%$ confidence test: $\alpha=0.05$.

Once we have calculated the defining limbs of each cluster, we can visualise them, as in Figure 6 . The visualisation is produced by taking the cluster means, and colouring the defining limbs in red. It should be noted that a defining limb can, in this sense, be very close to the mean - but with a different distribution around it. The legs of Cluster 16, for instance, are almost exactly at the mean, but with an atypically narrow distribution.

Such an ideal-type visualisation as Figure 6, combined with the Gaussiansample visualisation in Figure 5, can aid us in identifying the characteristic poses which constitute what Warburg describes as a Pathosformel - that is, a formula 

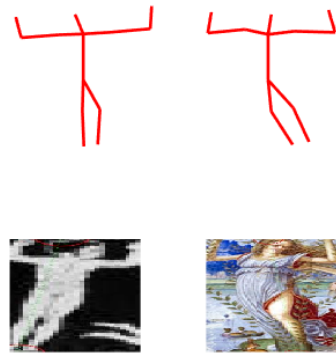

$-420$

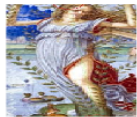

1475
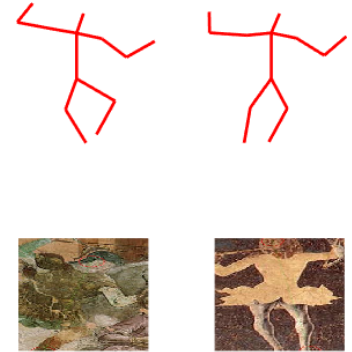

1490

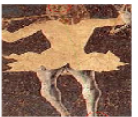

1470

Fig. 7: A selection of the dendrogram of cluster 8, showing related poses (and years) from antiquity and the Renaissance.

for the expression of emotion. One possible such instance, the cluster of figures with two raised arms, is shown in Figure 7.

In particular, we note the antithetical nature of the first two images - very similar poses, one from antiquity and one from the Renaissance, but with different emotional implications. The first shows a personified star being chased away by the sun. Rather than fleeing, the boy looks towards the sun defiantly, with open arms (see Figure $2 \mathrm{a}$ for the whole image). The second also represents a chased figure - Daphne, pursued by a desiring Apollo. After a long pursuit, Daphne prays to her father to destroy her beauty; and is turned into a tree. We thus see two morphologically similar poses but corresponding to two completely different notions of Pathos, defiance and desperation.

\subsection{Nachleben}

Having identified and investigated typical poses, we now turn our attention to Warburg's concept of Nachleben (see Section 1.2). In particular, we seek to identify some characteristic poses which are present in classical antiquity and in the Renaissance, and contrast them with those that seem to be novel inventions of the Renaissance.

We can see from Figure 8 that some forms seem to be present in antiquity (specifically, before 250 A.D) and experience a Nachleben (rebirth) in the Renaissance (after around 1250 A.D) - such as clusters 5, 12 and 15. Others (1, 4, $10,11)$ appear to be inventions of the Renaissance, without a classical precedent.

Unfortunately, our dataset comprises only one-third of the panels of the Bilderatlas, so we cannot make strong claims as to what is not present. However, it is surprising that we find no historical pattern of Nachleben for the Nymph (cluster 4). She is regarded by Warburg as one of the key figures of the Nachleben, who described her as a "pagan [i.e. pre-Christian] goddess in exile" [2]. Despite this, she seems quite rare in classical antiquity in our dataset; where the pose does appear, it generally relates to soldiers holding spears and shields, and not to female Nymphs. 
clust. 1

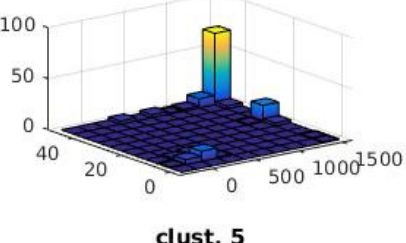

clust. 5

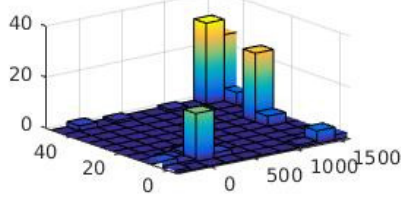

clust. 9

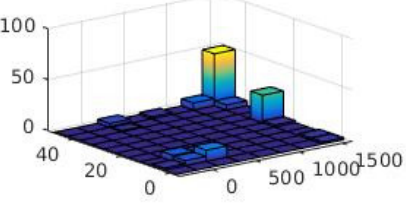

clust. 13

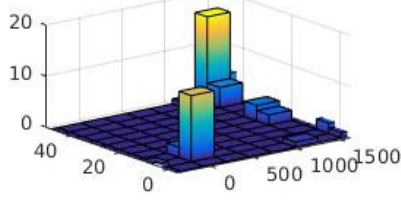

clust. 2

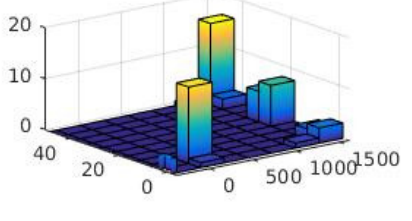

clust. 6

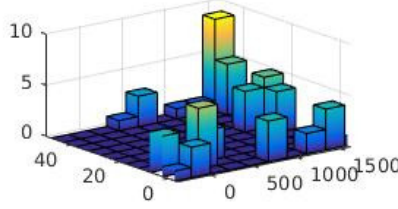

clust. 10

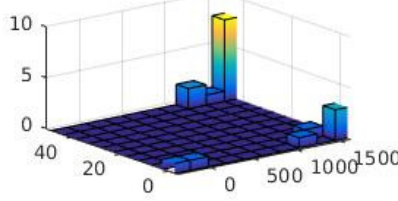

clust. 14

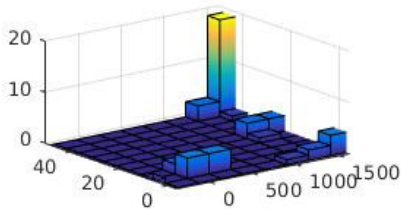

clust. 3

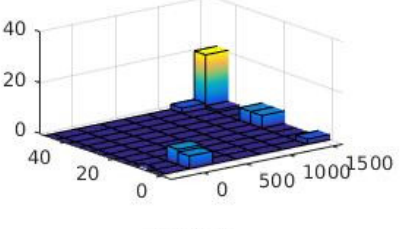

clust. 7

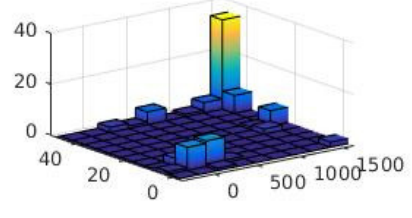

clust. 11

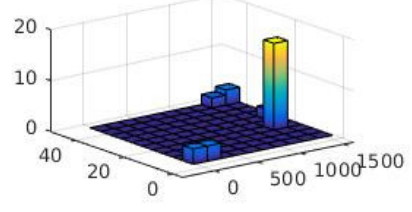

clust. 15

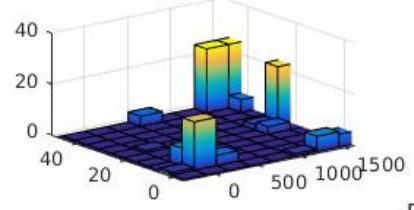

clust. 4

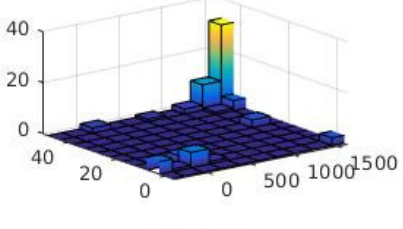

clust. 8

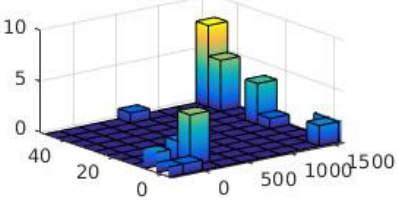

clust. 12

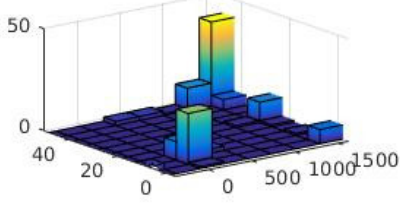

clust. 16

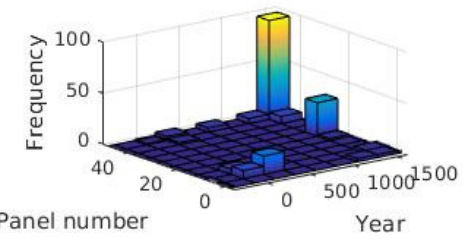

Fig. 8: 3D histograms showing the distribution through time (x-axis), and through panels (y-axis), of our 16 typical poses. Each time-period corresponds to 250 years. 


\section{Conclusions}

We have presented a methodology for extracting poses from a set of artwork of various styles, and for clustering and classifying these poses in 2D. From an art-historical point of view, we have given evidence to support some basic Warburgian notions: the presence of Pathosformeln, and their renewal from antiquity (the Nachleben). We have also made some more controversial suggestions: namely, that Pathosformeln can sometimes be characterised entirely by body pose (at least in the case of the Nymph), and that the Nymph does not seem to display an obvious Nachleben - at least, for our one-third subset of the Bilderatlas.

A clear limitation of our characterisation of the Pathosformel in terms of static poses is Warburg's own principle of antithesis - that similar poses are used for completely different actions. Whilst this static pose characterisation allows us to link similar antique and Renaissance forms together, we clearly ignore the inherent emotional ambiguity of static pose.

Indeed, we can propose a verifiable art-historical hypothesis based on Warburg's principle of antithesis. Artists might use visual cues for motion to give a static (image, not video) impression of dynamic pose. These visual movement cues might therefore suggest more precise emotional information.

Visual movement cues can be compared to Warburg's accessory forms [19]. In Warburg's discussion of Botticelli's Birth of Venus and Spring, he notes that Botticelli allows the Nymph to escape the emotional context of her classical legacy by manipulating "the surface mobility of inanimate accessory forms, draperies and hair" - after noting (in the same text) that the "most difficult problem in all art... is that of capturing images of life in motion" [17, p. 47].

\subsection{Further work}

With only one-third of the panels of the Bilderatlas digitised and annotated, a clear priority for future work is to annotate the remaining two-thirds - particularly because our most polemical claim (about the lack of Nachleben of the Nymph) refers to what is not present in our data (so far).

If the remaining two-thirds of the panels contain the same number of human bodies, we should finish with a set of 5,000 figures from painting and sculpture, each annotated with 2D human pose. This dataset would be of interest to the computer vision community (which currently lacks a large-scale pose dataset comparable to [18]).

The Art Historian David Freedberg claims that emotions are inseparable from the movement of the body [14] - following our hypothesis above on accessory forms as visual cues for movement (and therefore emotion), we might seek to investigate whether motion cues are already encoded in $2 \mathrm{D}$ pose, and in what way other visual cues for motion disambiguate the emotional implications of a pose. 


\section{Acknowledgments}

The authors would like to thank Isabella di Lenardo, Robin Osborne and John Robb, for their help and insights on the history of art and the body. Most of all, we are indebted to Franco Moretti, for his constant and wise guidance on this project.

\section{References}

1. Metaphor Lost and Found in Mnemosyne, pp. 70-109. Cornell University Press (2012)

2. Agamben, G., Cuspinero, A.: Ninfas (2010)

3. Angel, S.: The Mnemosyne Atlas and the Meaning of Panel 79 in Aby Warburg's Oeuvre as a Distributed Object. Leonardo 44(3), 266-267 (2011)

4. Argan, G.C.: Storia dell'arte italiana vol. 1. Sansoni, Florence, 2 edn. (1971)

5. Atkinson, A.P., Dittrich, W.H., Gemmell, A.J., Young, A.W.: Emotion perception from dynamic and static body expressions in point-light and full-light displays. Perception 33(6), 717-46 (jan 2004)

6. Atkinson, A.P., Tunstall, M.L., Dittrich, W.H.: Evidence for distinct contributions of form and motion information to the recognition of emotions from body gestures. Cognition 104(1), 59-72 (jul 2007)

7. Aviezer, H., Trope, Y., Todorov, A.: Body cues, not facial expressions, discriminate between intense positive and negative emotions. Science 338(6111), 1225-1229 (2012)

8. Becker, C.: Aby Warburg's Pathosformel as methodological paradigm. Journal of Art Historiography (2013)

9. Carneiro, G., da Silva, N.: Artistic image classification: an analysis on the printart database. Computer Vision-ECCV 2014 Workshops (2012)

10. Dortet-Bernadet, J., Wicker, N.: Model-based clustering on the unit sphere with an illustration using gene expression profiles. Biostatistics (2008)

11. Ekman, P.: An argument for basic emotions. Cognition \& Emotion 6(3-4), 169-200 (jan 1999)

12. Finin, T., Murnane, W., Karandikar, A., Keller, N., Martineau, J., Dredze, M.: Annotating named entities in Twitter data with crowdsourcing. In: Proceedings of the NAACL HLT 2010 Workshop on Creating Speech and Language Data with Amazon's Mechanical Turk. pp. 80-88. Association for Computational Linguistics (jun 2010)

13. Forster, K., Mazzucco, K.: Introduzione ad Aby Warburg e all'Atlante della memoria (2002)

14. Freedberg, D.: Empathy, motion and emotion. Wie sich Gefuehle Ausdruck verschaffen: Emotionen in Nahsicht f, 17-51 (2007)

15. Ginosar, S., Haas, D., Brown, T., Malik, J.: Detecting people in Cubist art. Computer Vision-ECCV $2014 \ldots$... (2014)

16. Johansson, G.: Visual perception of biological motion and a model for its analysis. Perception \& Psychophysics 14(2), 201-211 (jun 1973)

17. Johnson, C.D.: Memory, Metaphor, and Aby Warburg's Atlas of Images. Cornell University Press (2012)

18. Johnson, S., Everingham, M.: Clustered Pose and Nonlinear Appearance Models for Human Pose Estimation. BMVC (2010) 
19. Michaud, P.: Aby Warburg and the image in motion (2004)

20. Osborne, R.: The history written on the classical Greek body. Cambridge University Press (2011)

21. Poppe, R.: A survey on vision-based human action recognition. Image and Vision Computing 28(6), 976-990 (jun 2010)

22. Schoell-Glass, C.: Aby Warburg and Anti-semitism: Political Perspectives on Images and Culture. Wayne State University Press (2008)

23. Van Pelt, C., Sorokin, A.: Designing a scalable crowdsourcing platform. In: Proceedings of the 2012 international conference on Management of Data - SIGMOD '12. p. 765. ACM Press, New York, New York, USA (may 2012)

24. Warburg, A., Forster, K.W., Britt, D.: The Renewal of Pagan Antiquity: Contributions to the Cultural History of the European Renaissance, ed. Los Angeles: Getty Research Institute (1999)

25. Weber, M.: Objectivity in social science and social policy. The methodology of the social sciences 78 (1949)

26. Weinert, F.: Weber's Ideal Types as Models in the Social Sciences. Royal Institute of Philosophy Supplement 41, 73-93 (jan 2010) 